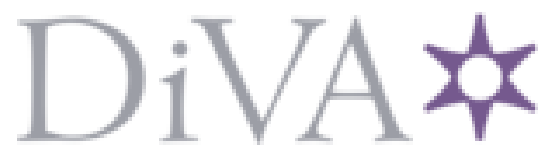

http://www.diva-portal.org

\title{
Postprint
}

This is the accepted version of a paper published in European Journal of Cardiovascular Nursing. This paper has been peer-reviewed but does not include the final publisher proof-corrections or journal pagination.

Citation for the original published paper (version of record):

Wahlström, M., Rydell Karlsson, M., Medin, J., Frykman, V. (2016)

Effects of yoga in patients with paroxysmal atrial fibrillation - a randomized controlled study..

European Journal of Cardiovascular Nursing, 16(1): 57-63

https://doi.org/10.1177/1474515116637734

Access to the published version may require subscription.

N.B. When citing this work, cite the original published paper.

Permanent link to this version:

http://urn.kb.se/resolve?urn=urn:nbn:se:shh:diva-2155 


\section{Effects of Yoga in patients with paroxysmal atrial fibrillation - a randomized controlled study}

Maria Nilsson RN, MA 12

Monica Rydell-Karlsson, $\mathrm{RN}, \mathrm{PhD} 12$

Jörgen Medin, RN, PhD 12

Viveka Frykman MD, $\mathrm{PhD}{ }^{1}$

${ }^{1}$ Karolinska institutet, Department of Clinical Sciences, Department of Cardiology, Danderyd Hospital, Stockholm, Sweden. This author takes responsibility for all aspects of the reliability and freedom from bias of the data presented and their discussed interpretation.

${ }^{2}$ Sophiahemmet University, Stockholm, Sweden. This author takes responsibility for all aspects of the reliability and freedom from bias of the data presented and their discussed interpretation.

Address for correspondence:

Maria Nilsson,

Hjärtforskningslaboratoriet

Danderyd Hospital

18288 Stockholm

Sweden

Telephone: $+46(0) 812357185$

Email: maria.nilsson@ds.se

Words: 2903 


\begin{abstract}
Background: Patients with atrial fibrillation often have an impaired quality of life (QoL).

Practicing yoga may decrease stress and have positive effects on mental and physical health. The aim of this study was to investigate whether yoga can improve QoL and decrease blood pressure and heart rate in patients with paroxysmal atrial fibrillation (PAF).

Methods: In this pilot study, eighty patients diagnosed with PAF were randomized to standard treatment (control group, $\mathrm{n}=40$ ) or standard treatment in combination with yoga (yoga group, $\mathrm{n}=40$ ) during a 12-week period. QoL, blood pressure and heartrate were evaluated at baseline and at the end of the study $(12(+2)$ weeks). EQ-5D VAS-scale and the two dimensions in SF-36 were used to evaluate QoL.
\end{abstract}

Results: At baseline there was a significant difference in QoL between the groups in EQ-5D VASscale $(\mathrm{p}=0.02)$ and SF-36 mental health score $(\mathrm{p}<0.001)$ in which the control group had higher scores. At the end of the study, the yoga group averaged higher SF-36 mental health scores. There was a significant difference between the two groups $(\mathrm{p}=0.016)$, but no differences in EQ-5D VAS- scale and physiological health score was seen between the two groups. At the end of the study, the yoga group had significantly lower heart rate $(\mathrm{p}=0.024)$ and systolic $(\mathrm{p}=0.033)$ and diastolic blood pressure $(\mathrm{p}<0.001)$ compared to the control group.

Conclusions: Yoga with light movements and deep breathing may lead to improved QoL, lower blood pressure and lower heart rate in patients with PAF compared to a control group. Yoga could be a complementary treatment method to standard therapy.

Keywords: paroxysmal atrial fibrillation, yoga, quality of life, blood pressure, heart rate. 


\section{Introduction}

\section{Atrial Fibrillation}

Atrial fibrillation is the most common heart rhythm disturbance associated with high mortality, morbidity and hospitalization (1). The most important risk factors for developing atrial fibrillation are increasing age and hypertension (2). Episodes of paroxysmal atrial fibrillation (PAF) can cause symptoms such as anxiety, stress and worry. Patients with PAF experience deterioration in health related quality of life (QoL) compared to the general population and patients with other cardiovascular diseases. This may potentially increase mortality, morbidity and hospitalization (36). PAF can also cause a decreased QoL among patients with a high arrhythmia burden and a high heart rate (7). To handle the emotional consequences that comes with AF the patients need practical tools (8). The standard treatment for PAF is medication, cardio version and ablation - to prevent severe complications, for example stroke. Reducing symptoms is the primary treatment goal for patients with PAF (1).

\section{Yoga}

Yoga practice consists of breathing techniques (pranayama) and meditation (dhyana) in combination with with various physical exercises (asanas) (9). Yoga is known to affect both the parasympathetic and sympathetic nervous systems. Practicing yoga can improve QoL (10) and as a result of its positive impact in reducing heart rate (11) and decreasing blood pressure (12), it may be useful in ameliorating various medical conditions. The effect of yoga however is under debate because many studies are small and not randomized $(13,14)$.

The aim of this study was to investigate whether yoga can improve QoL and decrease blood pressure and heart rate in patients with paroxysmal atrial fibrillation (PAF). 


\section{Methods}

This randomized pilot study was conducted at Danderyd Hospital in Stockholm, Sweden. The inclusion criterion was a diagnosis of PAF necessitating pharmacological treatment. Patients with difficulties understanding the Swedish language and patients with multiple concurrent medical conditions (i.e cancer, heart failure and renal failure with symptoms) or cognitive dysfunction were considered to have too many difficulties to perform yoga in a group session and were therefore excluded. The participants had either early or new diagnosis of PAF and should have been on medical treatment for PAF for at least three months. Eighty-eight patients with PAF were consecutive identified from medical records at the arrhythmia department's out-patient clinic and asked by telephone to participate. Eighty patients fulfilled the inclusion criteria and were accepted to participate in the study. Upon the baseline visit, the 80 patients were randomized; 40 patients were included in the yoga group and 40 patients in the control group (Figure 1). A permuted block randomization inspired by Polit and Beck, 2008, (15) was used. The patients were randomly assigned in blocks of five. The first five patients were allocated to either the yoga or the control group by blinded assignment. Randomization revealed the order of assignment for the next five patients (i.e. three to yoga and two to control). This procedure was repeated until one group had 40 respondents at which point the remaining patients were systematically allocated to the other group. All patients met the same research-nurse twice - first at baseline and finally after 12 weeks (within a 2 week visit window after intervention) at the end of the study - to assess QoL and hemodynamic parameters. Both visits (at baseline and at end of study) were held in the morning in the same room at the hospital for both groups. There was no change in environment for both groups' visits.

Medication treatments were collected from medical records at baseline and at the end of the study. 


\section{Ethics}

All participants provided verbal and written informed consent at baseline. The study was approved by the Ethics Committee of Stockholm, Sweden (DNR 2008/1983-31/2), Clinical Trial Gov Id: NCT01789372. The investigation conforms to the principles outlined in the Declaration of Helsinki.

\section{Intervention}

In this study we used a yoga form called Mediyoga (http://mediyoga.com). This is a therapeutic form of yoga evolved from Kundalini Yoga. It is a calm, meditative yoga based on deep breathing and is designed so that participants (if needed) may sit on a chair and exercise certain movements. The yoga program was specifically designed for people with cardiac diseases; it includes movements that stretch the muscles in the chest, which facilitates deep breathing and relaxes the body. Each session started with deep breathing for 5-10 minutes followed by three movements (back flex, back roll and Sat Kriya) that included two breathing techniques, subsequent meditation (ten minutes) and relaxation (ten minutes). A detailed description of the yoga program is available in a supplementary appendix. The intervention was performed under the instruction of an experienced yoga instructor. The instructor was not aware of changes in the standard medical therapy.

Participants performed yoga in group sessions once a week during the 12-week intervention period. Each yoga session took place in the hospital (physiotherapy clinic) and lasted one hour. Group session had a maximum of ten participants in each, who were also encouraged to practice yoga at home. To assist with self-instructed yoga, participants received a written program that included descriptions of movements together with a $\mathrm{CD}$. The control group was instructed not to perform any yoga during the intervention period. Both groups received standard treatment including medication, cardioversion and catheter ablation. 


\section{Endpoints}

The primary endpoint was difference in QoL after 12 weeks of yoga between the groups and within the groups. The secondary endpoints were blood pressure and heart rate from baseline to the end of the study.

\section{Quality of Life Assessments}

Two generic health-related QoL questionnaire, SF-36 (Short-Form Health Survey) $(16,17)$ and a VAS-scale from EQ-5D (EuroHRQoL-5D) $(18,19)$ were used.

SF-36 Short-Form Health Survey is a standardized, validated and reliable health-related QoL questionnaire $(16,17)$, which contains 36 questions frequently used in arrhythmia studies. These questions can be divided into eight domains and two dimensions: physical health and mental health. The two dimensions are analyzed into scores $0-100$ where 100 is the best possible state of QoL.

EQ-5D Visual Analog Scale (VAS-scale) is a part of EQ-5D and is also a standardized, validated and reliable scale $(18,19)$. This consists of a scale between $0-100$ where 100 is the best possible state of QoL.

The participants filled in the health related forms, SF-36 and EQ-5D VAS-scale at inclusion and at end of the study.

\section{Hemodynamic Assessments}

Systolic and diastolic blood pressure were measured on both visits. After sitting and resting for five minutes, the blood pressure was measured in the same arm with a calibrated automated blood pressure meter $\left(\mathrm{OMRON}^{\circledR}\right)$. Heart rate was also measured during one minute rest period. Statistical Analysis 
The variables in this study have been processed statistically using the data analysis program, SPSS version 12. Student's t-test (paired t-test) was used between the groups (yoga and control group) for blood pressure and heart rate. Mann Whitney U test was used in the analysis of QoL questionnaire SF-36 and EQ-5D VAS-scale and The Wilcoxon Signed Ranks test was used for within-group analyses. After controlling for outliers, an analysis of covariance (ANCOVA) was made to test if there were any significance differences in demographic data between the groups. The covariates we analysed were age, gender, hypertension and stroke. Demographic data is presented with mean, standard deviation (SD) or median, range, and percent. A significance level of $p<0.05$ was chosen.

\section{Results}

In the yoga group there were 33 patients who completed the intervention. Seven patients (17.5\%) - four men (10\%) and three women (7.5\%) - ceased to participating before the study period was completed. In the control group, 36 patients completed the study period; four male patients (10\%) ceased to participate. Clinical characteristics - age and sex between the two groups - are shown in Table 1. There were no significance differences between the yoga and the control group regarding age, gender, hypertension and stroke.

The use of medications is presented in Table 2. There was a trend of more use of antiarrhythmic medications and ACE inhibitors in the control group, but no significant changes in medication occurred over time. During the study period, two $(6 \%)$ respective three $(8 \%)$ patients performed an ablation in the yoga and control group. All completed the study. On average the participants in the yoga group performed ten yoga sessions (range 8-12) at the hospital. At the end of the study participants reported any yoga they may have conducted at home to the research nurse. At-home yoga was conducted at a median of 2 (range 1-4) sessions a week. 


\section{Quality of Life}

At baseline the yoga group estimated significantly lower QoL in EQ-5D VAS-scale as well as SF36 mental health scores compared to the control group, but no significant differences were observed between the groups regarding SF-36 physiological health scores (Table 3).

After 12 weeks the patients' estimated scores in EQ-5D VAS-scale had increased significantly (p $<0.001)$ in the yoga group whereas the control group experienced no change $(\mathrm{p}=0.622)$. The same pattern was seen in the estimated SF-36 mental health scores, with significant improvement in the yoga group $(\mathrm{p}<0.001)$ during the study that was not seen in the control group $(\mathrm{p}=0.782)$.

In the subscales of physical health in SF-36 scores, no significant difference was seen between or within the groups at the end of the study.

\section{Hemodynamic Assessments}

There were two different patients, one at baseline and one at the end of the study, which had atrial fibrillation according to ECG. At baseline there were no differences between the two groups in heart rate or systolic and diastolic blood pressures. After 12 weeks of yoga, heart rates were significantly lower in the yoga group compared to the control group $(\mathrm{p}=0.024)$ as well as systolic $(\mathrm{p}=0.033)$ and diastolic $(\mathrm{p}<0.001)$ blood pressures (Table 4).

\section{Discussion}

This is a pilot study. As our knowledge this is one of the largest randomized studies evaluating the effect of yoga in patients with PAF. Our results show that yoga significantly improves QoL and that their increased comfort allows them to more easily tolerate their conditions. Decreasing blood pressure and resting heart rate may give patients a feeling of security. Decreasing suffering and helping patients to feel safe are critical elements of nurses' roles as regards their patients (20). 
When patients with atrial fibrillation are compared with healthy controls, the general population and patients with coronary heart disease in sinus rhythm, they present with an impaired QoL (4). One of the most important treatment goals in atrial fibrillation is to reduce symptoms and improve QoL (21). The result of our study is that a 12-week yoga program can improve QoL in patients with PAF. This has also been reported by previous studies that indicated that yoga can contribute to patients' wellbeing and reduce episodes of atrial fibrillation (22). Yoga has also been reported to increase self-esteem in social QoL (11) and improve QoL in individuals with congestive heart failure (10). A recent study also reported data that yoga can improve the QoL, anxiety and depression in patients with PAF (22). Our results may bring access to a complementary treatment that allows patients with PAF to achieve a healthier life, improve their sociability and increase their QoL.

Although we performed a randomized study, the two groups were not completely balanced; the yoga group estimated a lower QoL regarding EQ5D Vas-scale and SF-36 mental health at baseline. In our study we had relatively more women and patients with a previous stroke/TIA in the yoga group and in earlier studies women with PAF estimate poorer QoL than men $(23,24)$ and patients who have suffered a stroke estimate poorer QoL than the general population (25). This asymmetry could influence our results at baseline.

Quality of life can be difficult to measure given the various factors that may impact it. Factors that can contribute to quality of life may occur at different times, which can affect quality of life measurements (26). The measurements were equal for both groups in this study and therefore should have no influence on the results.

In our study heart rate as well as systolic and diastolic blood pressure was significantly lower in the yoga group compared to the control group, in which no changes in blood pressure were observed. This effect on blood pressure and heart rate may also be another explanation to 
improved QoL among patients practicing yoga. Hypertension is an important risk factor for atrial fibrillation related complications. Many of these patients have insufficiently regulated blood pressure, which may cause unnecessary cardiovascular events $(2,27)$ including the development of PAF and recurrent atrial fibrillation (8). Decreasing blood pressure may lead to a reduced risk for bleeding in treatment with anticoagulants. Studies with yoga describe similar effects on blood pressure as we do $(11,12)$. Literature recommends slow deep breathing to lower blood pressure (14), and we used a yoga form based on slow deep breathing. It seems that patients who have increased heart rate and blood pressure may have a deteriorated QoL $(27,28)$.

At the same time, high heart rates are relatively common and may lead to pronounced symptoms and eventually development of a tachycardiomyopathy (29). Decreasing blood pressure and heart rate with yoga appears to reduce complications with other medical conditions such as stroke and heart failure in patients with PAF, although this requires more careful examination.

It is becoming more common for patients with arrhythmia to use complementary alternative medicine, including yoga (30). It is therefore becoming more and more significant to evaluate the effects of yoga in providing safe treatments for those patients.

It is important to improve QoL in patients with PAF as well as to decrease their mortality and hospitalization (31). During our study the yoga group substantially improved their estimated QoL according to EQ-5D VAS and SF-36 mental health, in contrast to the control group in which no improvement was seen. One important reason for this is that yoga may give patients with PAF a method to gain some self-control over their symptoms. As the incidence and cost of PAF treatment increases in society, yoga may be a complementary treatment strategy to improve PAF care. 


\section{Limitations}

Although this is one of the largest randomized study evaluating yoga in PAF, it included a rather small number of included subjects. That may have contributed largely to the differences between the study groups at baseline. A larger study would be needed to confirm the effects of yoga in patients with PAF. We also included patients with PAF irrespective of their symptoms. The effect may be more pronounced if the study only includes patients with a symptomatic arrhythmia. Episodes of atrial fibrillation may lower the estimated QoL. The numbers of episodes of atrial fibrillation and symptoms were not reported in this study. This exclusion can influence the result. The participants in the intervention group may have had more episodes of atrial fibrillation, and those episodes could have produced to more symptoms in the intervention group than were observed in the control group. This may influence the results in QoL assessments at baseline. Another limitation of this randomized study is that the group therapy of yoga can have a placebo effect and increase QoL.

SF-36 and EQ-5D VAS-scale are general quality of life questionnaires and include no questions about symptoms. A more specific form to evaluate QoL for patients with PAF would be more appropriate.

Although we found good effects on physical markers in the yoga group, such as decreased heart rate and blood pressure, our understanding of this positive effects may benefit to further research. The further research should, for example, also include bigger sample size, comparing different interventions (for example relaxation technique) and measuring the episodes of atrial fibrillation over time.

\section{Conclusion}

Yoga with light movements and deep breathing may lead to improved QoL, lower blood pressure and decreased heart rate in patients with PAF compared to a control group not performing yoga. Yoga could be a complementary treatment method to standard therapy. 


\section{Implications for practice}

- This is the largest randomized study with yoga and paroxysmal atrial fibrillation.

- Yoga with light movements and deep breathing leads to improved QoL, lower blood pressure and heart rate in patients with PAF compared to a control group.

- The yoga program we used in this study is suitable for patients with PAF as it involves movements, deep breathing and meditation suitable for practice at any age. It uses the same type of techniques as other yoga forms: long deep breaths, various movements and meditation.

- We believe that this form of yoga could be implemented due to its simplicity and can be carried out generally in patients with PAF.

- Yoga can be a complementary treatment method to standard therapy.

\section{Acknowledgements}

The authors thank the yoga instructor Göran Boll, Jenny Rasck, BMA, and the staff who were involved at Danderyd Hospital for support in this study.

\section{Funding Sources}

This work was supported by Fondmedel 176 KI, Stockholm, Sweden

The authors declares that there is no conflict of interest 


\section{References}

1. Camm A, Kirchhof P, Lip G, Schotten U, Savelieva I, Ernst S, et al. Guidelines for the management of atrial fibrillation. The Task Force for the Management of Atrial Fibrillation of the European Society of Cardiology (ESC). Eur Heart J 2010; 19: 2369-429.

2. Huxley RR, Lopez FL, Folsom AR, Agarwal SK, Loehr LR, Soliman EZ, et al. Absolute and attributable risks of atrial fibrillation in relation to optimal and borderline risk factors: the Atherosclerosis Risk in Communities (ARIC) Study. Circulation 2011; 123: 1501-8.

3. Thrall G, Lane D, Carroll D, Lip GY. Quality of life in patients with atrial fibrillation: a systematic review. Am J Med 2006; 119: 448- 9.

4. McCabe PJ. Psychological distress in patients diagnosed with atrial fibrillation: the state of the science. J Cardiovasc Nurs 2010; 25: 40-51.

5. Suzuki S, Kasanuki H. The influences of psychosocial aspects and anxiety symptoms on quality of life with arrhythmia: investigation in paroxysmal atrial fibrillation. Int J Behav 2004; 11: 104-9.

6. McCabe PJ, Barnason SA. Illness perceptions, coping strategies and symptoms contribute to psychological distress in patients with recurrent symptomatic atrial fibrillation. J Cardiovasc Nurs 2011; 27: 431-44.

7. van den Berg MP, Hassink RJ, Tuinenburg AE, van Sonderen EF, Lefrandt JD, de Kam PJ, et al. Quality of life in patients with paroxysmal atrial fibrillation and its predictors: importance of the autonomic nervous system. Euro Heart J 2001; 22: 247-53. 
8. Ekblad H, Rönning H, Fridlund B, Malm D. Patients' well-being: experience and actions in their preventing and handling of atrial fibrillation. Eur J Cardiovasc Nurs 2013; 12: 132-9.

9. Corby JC, Roth WT, Zarcone VP, Kopell BS. Psychophysiologic correlates of the practice of tantric yoga meditation. Arhives of general psychiatry 1978; 35: 571-7.

10. Howie-Esquivel J, Lee J, Collier G, Mehling W, Fleischmann K. Yoga in heart failure patients: a pilot study. J Card Fail 2010; 16: 742-9.

11. Veerabhadrappa SG, Baljoshi VS, Kanapure S,Herrur A, Patil S, Ankad RB, et al. Effect of yogic bellows cardiovascular autonomic reactivity. J Cardiovasc Dis res 2011; 2: 223-7.

12. Cohen DL, Bloedon LT, Rothman R, Farrar JT, Galantino ML, Volger S, et al. Iyengar yoga versus enchanced usual care on blood pressure in patients with pre hypertension to stage I hypertension: a randomized controlled trial. Evid Based Complement Alternat Med 2011; [Epub 2011 Feb14],10.1093/ecam/nep130.

13. Kwong JS, Lau HL, Yeung F, Chau PH. Yoga for secondary prevention of coronary heart disease (review). Cochrane Database Syst Rev 2015 1; 7: CD009506.

14. Brook R, Appel L, Rubenfire M, Ogedegbe G, Bisognano JD, Elliott WJ, et al. Beyond Medications and diet: Alternative approaches to lowering blood pressure: A scientific statement from American Heart Association. Hypertension 2013; 61: 1360-83. 
15. Polit, DF, \& Beck CT. (2008). Nursing research: Generating and assessing evidence for nursing practice $\left(9^{\text {th }}\right.$ ed.). Philadelphia: Lippincott Williams \& Wilkins.

16. Ware JE, Sherbourne CD. The MOS 36-item Short-Form Health Survey (SF-36). Med Care 1993; 30: 473-83.

17. Sullivan M, Karlsson J, Ware J. The Swedish SF-36 health survey-I. Evaluation of data quality, scaling assumptions, reliability and construct validity across general populations in Sweden. Soc Sci Med 1995; 41: 1349-58.

18. The EuroQol Group Euro Qol—a new facility for the measurement of health related quality of life. Health Policy 1990; 16: 199-208.

19. Rabin R, Charro F.EQ-5D: a measure of health status from the EuroQol Group. Ann Med 2001; 33: 337-43.

20. International Council of Nurses. ICN: s, International Council of Nurses, codes of ethics for nurses, www.icn.ch, 2015.

21. Schron E, Friedmann E, Thomas SA. Does health-related quality of life predict hospitalization or mortality in patients with atrial fibrillation? J Cardiovasc Electrophysiol 2014; 25: 23-8.

22. Lakkireddy D, Atkins D, Pillarisetti J Ryschon K, Bommana S, Drisko J, et al. Effects of yoga on arrhythmia burden, anxiety, depression, and quality of life in paroxysmal atrial fibrillation. $J$ Am Coll Cardiol 2013; 19: 1177-82. 
23. Reynolds MR, Lavelle T, Essebag V, Cohen DJ, Zimetbaum P. Influence of age, sex, and atrial fibrillation recurrence on quality of life outcomes in a population of patients with new-onset atrial fibrillation: the Fibrillation Registry Assessing Costs, Therapies, Adverse events and Lifestyle (FRACTAL) study. Am Heart J. 2006; 152: 1097-103.

24. Dares N, Nieuwlaat R, Vardas PE, Andresen D, Lévy S, Cobbe S, et al. Gender-related differences in presentation, treatment, and outcome of patients with atrial fibrillation in Europe: a report from the Euro Heart Survey on Atrial Fibrillation. J Am Coll Cardiol 2007; 49: 572-77.

25. Vlajinac H, Marinkovi J, Maksimovic M, Kocev N, Vasiljevic N, Backovic D, et al. Healthrelated quality of life among patients with symptomatic carotid disease. Postgrad Med J 2013; 89: 8-13.

26. Garratt A, Schmidt L, Mackintosh A, Fitzpatrick R. Quality of life measurement: bibliographic study of patient assessed health outcome measures. BMJ. 2002; 324: 1417.

27. Lau YF, Yiu KH, Siu CW, Tse HF. Hypertension and atrial fibrillation: epidemiology, pathophysiology and therapeutic implications. J Hum hypertens 2012; 26: 563-926.

28. Groenvald HF, Crijns HJ, Van den Berg MP,Van Sonderen E, Alings AM, Tijssen JG, et al.; RACE-II-investigators. The effect of rate control on quality of life in patients with permanent atrial fibrillation: data from the RACE II (Rate Control Efficacy in Permanent Atrial Fibrillation II) study. J Am Coll Cardiol 2011; 58: 1795-1803. 
29. Umana E, Solares CA, Alpert MA. Tachycardia-induced cardiomyopathy. Am J Med. 2003; 114: $51-5$

30. Brenyo A, Aktas MK. Review of complementary and alternative medical treatment of arrhythmias. Am J Cardiol. 2014; 113: 897-903.

31. Steg G, Alam S, Chiang C-H, Gamra H, Goethals M, Inoue H, et al. ; RealiseAF investigators. Symptoms, functional status and quality of life in patients with controlled and uncontrolled atrial fibrillation: data from the RealiseAF cross-sectional international registry. Heart 2012; 98: 195201. 